\title{
Results of thoracotomy in osteogenic sarcoma with pulmonary metastases
}

\author{
S R Carter, R J Grimer, R S Sneath, H R Matthews
}

\begin{abstract}
Background Resection of pulmonary metastases may be followed by long term survival and now that it is an accepted method of treatment for patients with osteogenic sarcoma indicators of favourable prognosis are needed to aid the assessment of suitability for resection. This study compares the survival rates of patients who did and did not undergo resection of their pulmonary metastases and relates them to prognostic indicators.
\end{abstract}

Methods The study population was the 43 patients with osteosarcoma who developed pulmonary metastases out of the 11 patients with osteosarcoma treated by the Birmingham bone tumour treatment service during 197783. All patients who developed metastases confined to the lungs were considered for resection, thoracotomy being advised for all patients (provided that they were fit enough) who had metastases thought to be resectable even if they were multiple.

Results Of the 18 patients who did not have a thoracotomy, 15 died of disseminated disease after a mean interval of eight months; one patient died of cardiomyopathy and two are alive after 26 and eight months. Of the 25 patients who underwent thoracotomy in an attempt to resect metastases, three were found to have inoperable disease and died after a mean interval of 5.4 months from thoracotomy. Overall, after thoracotomy (repeated if necessary) there was a $20 \%$ survival at five years from the first thoracotomy. When survival was assessed with respect to the disease free interval and the number and bilaterality of the metastases no significant relationships were found. There was, however, a significant relation between survival and the position of metastases, patients with metastases confined to one lobe of the lung having a mean survival of 29.5 months, compared with 13.7 months in patients with disease in more than one lobe.

Conclusion Thus patients who had a thoracotomy survived longer from the time of diagnosis of pulmonary metastasis than those not undergoing thoracotomy; metastases confined to one lobe predicted a better prognosis.
The survival of patients with osteosarcoma is improving, ${ }^{1}$ principally because of the increasing use of chemotherapy. The disease free survival rate at five years is now greater than $50 \%$. Of patients developing metastases, $70 \%$ or more have metastases in the lung. ${ }^{23}$ Patients who die from metastatic spread of malignant disease to the lungs often have no other disseminated disease $e^{4}$ and the pulmonary deposits are often resectable. These findings suggest that resection of metastases may be curative. The first reported resection of a pulmonary metastasis was by Torek in $1930 .{ }^{5}$ The subsequent report by Barney and Churchill in $1939^{6}$ of a patient surviving after resection of pulmonary metastases from a renal cell carcinoma was followed by many reports of long term survival after resection of pulmonary metastases. Much effort has been directed towards the identification of favourable prognostic indicators in such patients, including the disease free interval, the tumour doubling time, the number of metastases, and tumour type and grade.

Before resection of pulmonary metastases from osteogenic sarcoma became an accepted method of treatment the three year survival from the time of diagnosis of pulmonary metastases was $5 \% .^{7}$ Spanos $^{8}$ reported a three year survival of $65 \%$ in a series of 30 patients undergoing pulmonary resection and a five year survival of $28 \%$. Putnam ${ }^{2}$ with a series of 39 patients and $\mathrm{Han}^{9}$ with a series of 111 patients who received chemotherapy in addition to resection reported a five year survival rate of $40 \%$.

Since 1977 patients under the care of the Birmingham bone tumour treatment service with pulmonary metastases from osteogenic sarcoma have been referred immediately to a thoracic surgery unit for assessment of suitability for resection. This paper compares the survival of patients who underwent resection of their metastases with that of those who did not undergo resection and looks at the response in relation to prognostic indicators.

\section{Methods}

The bone tumour treatment service at the Royal Orthopaedic Hospital had 111 new cases of osteogenic sarcoma referred to it between its inception in 1977 and December 1983, when the review was closed to allow for a five year follow up after its completion. The tumours were surgically staged according to the method of Enneking. ${ }^{10}$ Osteogenic sarcoma is a high grade malignant tumour and is therefore 
Figure 1 Treatment and outcome of the 111 patients with osteogenic sarcoma seen in the

Birmingham bone tumour treatment service up to 1984.

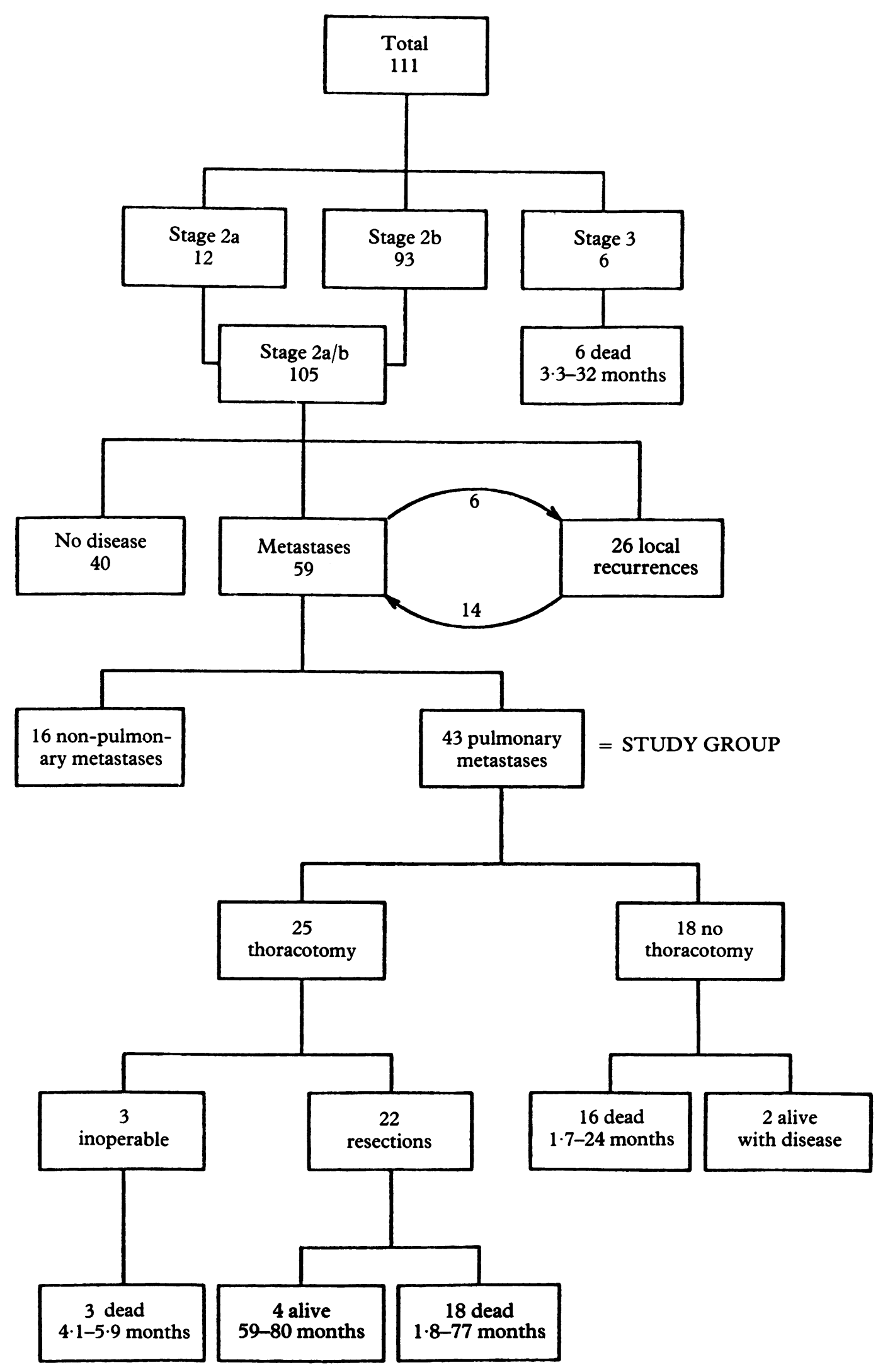

classified as a stage 2 tumour. In 12 patients the tumour was confined to the bone and was thus classified as stage $2 a$, and in 93 the disease affected two contiguous anatomical compartments without distant metastases and was classified as stage $\mathbf{2 b}$. Six patients had distant metastases at presentation (stage 3 ). The stag- ing was based on the results of radiographs, isotope bone scans, and pathological findings at the time of surgery.

The mean age of the patients at presentation was $20 \cdot 1$ (range 6.3-66) years; 71 were male. The average duration of symptoms was 13.1 weeks. The site of the primary tumour was the 
femur in 56 patients; tibia in 31 ; humerus in 16 ; fibula in three; ilium in two; and ribs, ischium, and clavicle in one patient each.

The surgical treatment of the primary tumour was amputation for 16 patients, excision for six, and endoprosthetic replacement for 89 .

All patients received chemotherapy at the time of surgical treatment. Fifty seven patients received 11 weeks' high dosage methotrexate of $3-7.5 \mathrm{~g} / \mathrm{m}^{2}$ body surface area with leucovorin rescue. Twenty nine had vincristine, doxorubicin (Adriamycin) and low dose methotrexate. The remaining patients had various other chemotherapy regimens.

The patients were reviewed regularly after treatment, initially at three monthly intervals until two years after completion of treatment and thereafter six monthly. At each attendance posteroanterior and lateral radiographs of the chest were performed routinely.

All patients who developed metastases confined to the lungs were referred to a thoracic surgeon for assessment with a view to possible resection. As it was uncertain whether resection of bilateral metastases is worth while, thoracotomy was advised for all patients in whom the metastases, even if multiple, were thought to be capable of total resection, provided that the patient was fit enough to undergo the procedure.

After thoracotomy and resection of metastases 13 patients were given adjuvant chemotherapy and 10 patients did not receive chemotherapy.

The results were assessed by standard statistical methods. Means were compared by

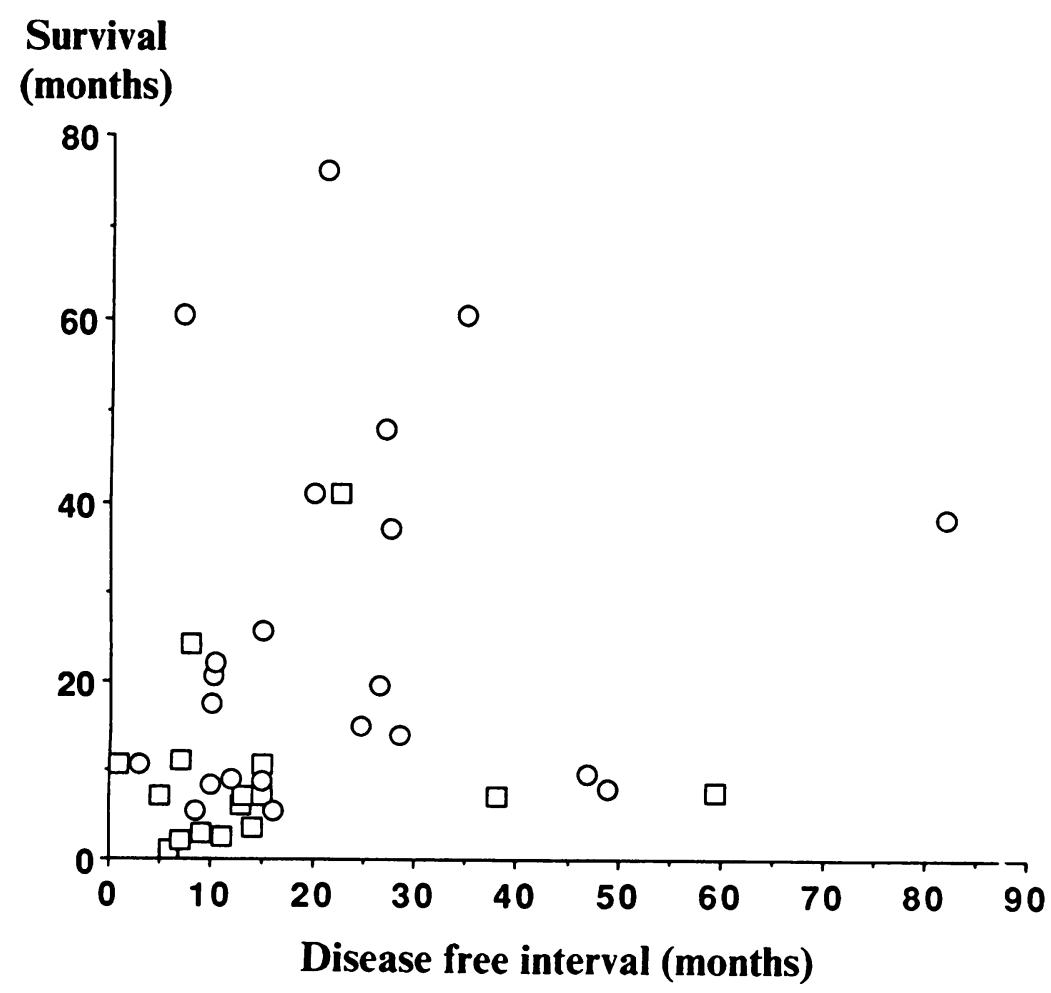

Figure 2 Random distribution of disease free interval in relation to duration of the patient's survival from the discovery of pulmonary metastases. $\square$ Patients who did not undergo resection of the pulmonary metastases; $O$ patients whose pulmonary metastases were resected.
Student's $t$ test and frequencies by the $\chi^{2}$ test. Cumulative survival was calculated by the method described by Armitage. ${ }^{11}$

\section{Results}

The mean period of follow up from diagnosis was $7 \cdot 4$ (range 5.2-11.6) years. In the 105 patients with stage 2 disease metastases were found in $59(55 \%)$. Of these patients, $43(73 \%$ of those with metastatic disease) had developed pulmonary metastases alone; these 43 patients form the study group. There was no difference in the incidence of metastases between patients who had had an endoprosthetic replacement and those who had had an amputation. Twenty five patients proceeded to thoracotomy and 22 underwent wedge resection of one to six individual metastases (fig 1).

\section{PATIENTS UNSUITABLE FOR THORACOTOMY}

Eighteen of the 43 patients with pulmonary metastases were considered unsuitable for thoracotomy. Fifteen of these patients died of disseminated disease an average of eight (range 1.7-24) months after the onset of the metastases. Of the remaining three patients, one is alive 26 months after the development of the pulmonary metastases, though a local recurrence has developed also, and one is alive after eight months, 73 months after the original diagnosis of osteosarcoma. The third died as a result of doxorubicin cardiotoxicity.

\section{PATIENTS SUITABLE FOR THORACOTOMY:}

SURGICAL PROCEDURES

Twenty five patients underwent thoracotomy. At surgery the disease was found to be too extensive for any attempt at resection in three patients. In the remaining 22 patients the resection resulted in complete removal of clinically apparent disease. Eleven resections were performed for a single metastasis and 11 for multiple metastases. In 14 patients the metastases were confined to a single lobe of the lung whereas eight had metastases in more than one lobe. In the four patients with bilateral disease resections were performed through a median sternotomy.

Two patients had two thoracotomies and two had three thoracotomies for recurrence of metastases. The mean time between the first and second thoracotomy was $15 \cdot 7$ (range 7-24) months and between the second and third was 17.5 (range 8-33) months. Of the four patients who underwent multiple thoracotomies, one is alive and free of disease 89 months after the first thoracotomy (39 months after the third thoracotomy). The other three patients have died an average of 51 months from the onset of their pulmonary metastases.

\section{OUTCOME}

Of the 25 patients who underwent thoracotomy, four are still alive (one after repeated thoracotomies), a median of 69 (range 59-80) months from the diagnosis of the first pulmonary metastasis. Twenty one patients have died. The three with inoperable metastases died a median of $5 \cdot 4(4 \cdot 1-5 \cdot 9)$ months 


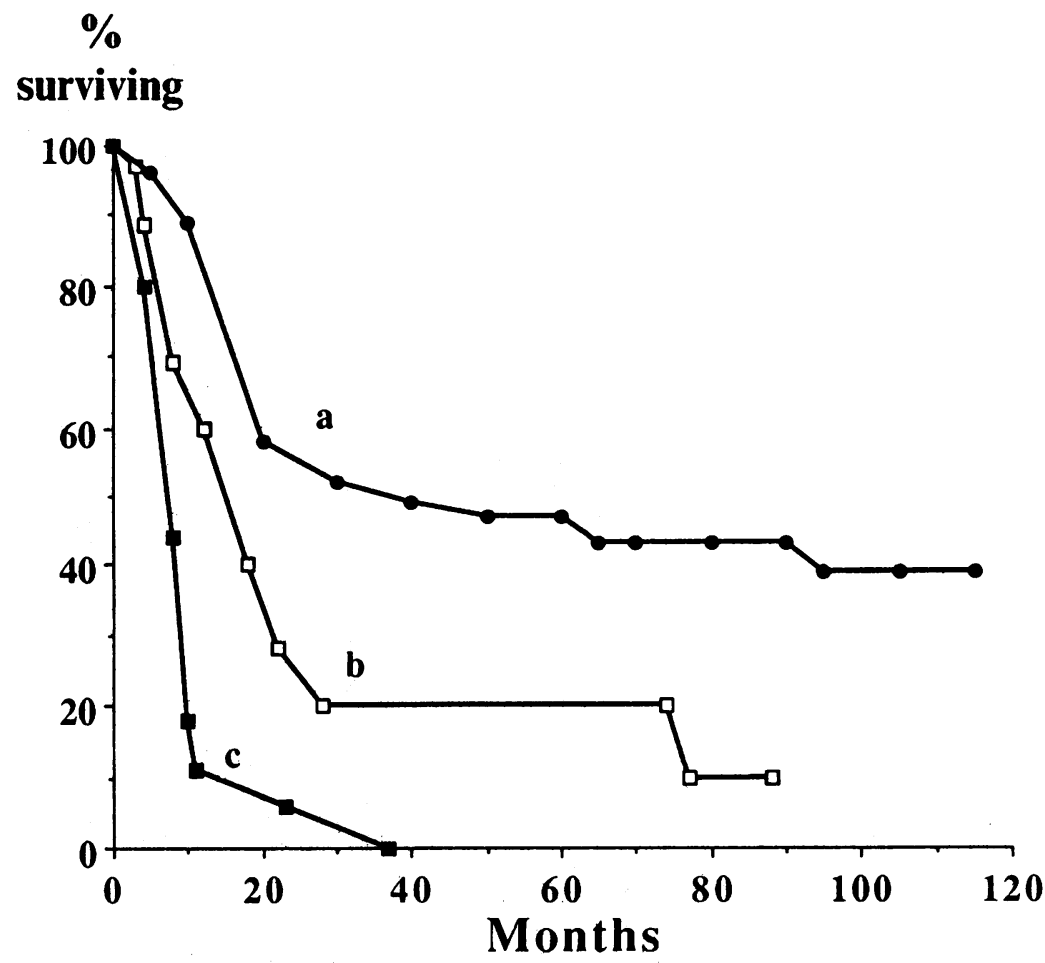

Figure 3 Cumulative survival curves for patients with osteosarcoma treated up to the erd of 1983. Curve $a$-survival of all 111 patients from diagnosis of osteosarcoma; $b$ survival from diagnosis of pulmonary metastases of the 25 patients who underwent thoracotomy; $c$-survival from diagnosis of pulmonary metastases of the 18 patients who did not have a thoracotomy.
The number of pulmonary metastases resected showed no significant relation to the time taken for the initial pulmonary metastases to appear, or to survival from the appearance of the metastases. There was also no significant relation between survival and whether the pulmonary metastases were unilateral or bilateral, or between the disease free interval and survival (fig 2).

\section{Discussion}

In recent years a change in the distribution of metastatic disease arising from osteosarcoma appears to have taken place, with a decrease in the proportion of pulmonary metastases ${ }^{12}$ but an increase in the number of bony metastases. ${ }^{13}$ This may be a result of a natural alteration in the disease process with time ${ }^{14}$ but is more likely to be due to improvements in adjuvant chemotherapy, ${ }^{15}$ resulting in longer survival or to more sensitive diagnostic aids such as computed tomography.

Patients who died of their disease after thoracotomy must have had other metastases present at the time of the resection that were not detected. Identifying the extent of the disease before resection is therefore essential. Gundry $^{16}$ concluded that the presence of tumour microfoci in the pulmonary resection specimens was associated with an increased recurrence rate. Detailed histological assessment of the resected specimen may improve prognostic accuracy.

In patients who develop metastases, the adjuvant chemotherapy must be considered a failure. ${ }^{1517}$ Patients given chemotherapy for metastatic osteogenic sarcoma did not appear to survive longer than those not receiving chemotherapy in this series, despite the hope that it would control micrometastatic disease. Possibly those patients who have developed metastatic disease after receiving chemotherapy are not likely to respond to further chemotherapy even though different drugs may be administered. Thus the likely benefit of chemotherapy should be weighed against the morbidity and decrease in quality of life of these patients. A controlled clinical trial is needed to assess the benefit and risk more fully.

The appearance of pulmonary metastases after surgical treatment of the primary disease and chemotherapy implies that micrometastatic disease was present but undetectable before excision of the primary tumour. The time taken for these metastases to become apparent may be an indication of the prognosis. An initial disease free interval of one year or less has been considered a worse prognostic indicator than a disease free interval of more than one year. ${ }^{18} 19$ There was no relation between the disease free interval and survival in our study either for those who did not have a thoracotomy or for those who had a thoracotomy.

Our results (20\% five year survival) differ from those of studies from both the United States and the United Kingdom, which predict a five year survival ranging from $30 \%$ to $57 \%$ in patients who have had resection of pulmonary
Occurrence of further metastases

Of the 25 patients who had a thoracotomy, 21 developed further metastases. The mean time taken for this second metastasis to be diagnosed was 11 months. This interval was not related to the initial disease free period. 
metastases. ${ }^{12917}$ Many of those patients were assessed regularly at monthly intervals with the aid of computed tomography, which can show smaller pulmonary metastases than conventional chest radiography. ${ }^{20}$ The use of computed tomography may allow earlier detection of the presence and the site of metastatic disease, which may account for the improved five year survival in these studies.

We found that survival was related not to the number of pulmonary metastases but to the distribution of the pulmonary metastases, patients with metastases confined to one lobe of the lung surviving longer than those with widespread metastases. Reports on previous series do not give details of distribution, though they do comment on the number of metastases. A patient with a single metastasis obviously has only one lobe affected and this may be reflected in their longer survival. Multiple thoracotomies appear to result in increased survival in patients with a further recurrence confined to the lungs. The results reported here are based on a follow up similar in duration and in the number of patients to those in other reported series. ${ }^{18915}$.

We conclude from this study that thoracotomy may increase survival after the development of pulmonary metastases, though it is unlikely to cure the patient. Our results may be improved by more careful review of the patients, with the use of computed tomography, to attempt to detect metastases at an earlier stage and to identify those with disease confined to one lobe of the lung.

1 Goorin AM, Delorey MJ, Lack EE, et al. Prognostic significance of complete surgical resection of pulmonary metastases in patients with osteogenic sarcoma: Analysis of 32 patients. J Clin Oncol 1984;2:425-31.

2 Putnam JB, Roth JA, Wesley MN, Johnston MR, Rosen- berg SA. Survival following aggressive resection of pulmonary metastases from osteogenic sarcoma: analysis of prognostic factors. Ann Thorac Surg 1983;36:516-23.

3 Giuliano AE, Feig S, Eilber FR. Changing metastatic patterns of osteosarcoma. Cancer 1984;54:2160-4.

4 Farrell JT. Pulmonary metastases: a pathologic, clinical and roentgenologic study based on 78 cases seen at necropsy. Radiology 1935;24:444-51.

5 Torek F. Removal of metastatic carcinoma of the lung and mediastinum. Arch Surg 1930;21:1416-24.

6 Barney JD, Churchill ED. Adenocarcinoma of the kidney with metastases to the lung cure by nephrectomy and lobectomy. J Urol (Paris) 1939;42:269-76.

7 Marcove RC, Mike V, Hajek JV, Levin AG, Hutter RVP. Osteogenic sarcoma under the age of twenty one, a review of one hundred and forty five operative cases. J Bone Joint Surg $(\mathrm{Am})$ 1970;48:411-23.

8 Spanos PK, Payne WS, Ivins JC, Pritchard DJ. Pulmonary resection for metastatic osteogenic sarcoma. J Bone Joint Surg ( Am) 1976;58:624-8.

9 Han M-T, Telander RL, Pairolero PC, Payne WO, Sim FH, Pritchard DJ. Aggressive thoracotomy for pulmonary metastatic osteogenic sarcoma in children and young adolescents. J Pediatr Surg 1981;16:928-33.

10 Enneking WF. Musculoskeletal tumour surgery. New York Churchill Livingstone, 1983:81-96.

11 Armitage P. Statistical methods in medical research. Oxford: Blackwell, 1980:410-4.

$12 \mathrm{Jaffe}$ N, Smith E, Ableson HT, Frei E. Osteogenic sarcoma: alterations in pattern of pulmonary metastases with adjuvant chemotherapy. $J$ Clin Onccl 1983;1:251-4.

13 Goldstein H, McNeil BJ, Zufall E, Jafee N, Treves S Changing indications for bone scintigraphy in patients with osteosarcoma. Radiology 1980;135:177-80.

14 Taylor WF, Ivins JC, Dahlin DC, Edmonson JH, Pritchard DJ. Trends and variability in survival from osteosarcoma. Mayo Clin Proc 1978;53:695-700.

15 Simon MA. Cause of increased survival of patients with osteosarcoma: current controversies. J Bone Joint Surg ( $\mathrm{Am}$ ) 1984;66A:306-10.

16 Gundry SR, Coran AG, Lemmer J, Wesley JR, Hutchinson $R$. The influence of tumor microfoci on recurrence and survival following pulonary resection of metastatic osteogenic sarcoma. Ann Thorac Surg 1984;38:473-7.

17 Al-Jilaihawi AN, Bullimore J, Mott M, Wisheart JD. Combined chemotherapy and surgery for pulmonary metastases from osteogenic sarcoma. J Cardiothorac Surg 1988;2:37-42.

18 Telander RL, Pairolero PC, Pritchard DJ, Sim FH, Gilchrist GS. Resection of pulmonary metastatic osteogenic sarcoma in children. Surgery 1978;84:335-41.

19 Huth JF, Holmes EC, Vernon SE, Callery CD, Ramming KP, Morton DL. Pulmonary resection for metastatic sarcoma. Am J Surg 1980;140:9-15.

20 Chang AE, Schaner EG, Conkle DM, Flye MW, Doppman JL, Rosenberg SA. Evaluation of computed tomography in the detection of pulmonary metastases. A prospective study. Cancer 1979;43:913-6. 